\title{
Quantity in Quantum Mechanics and the Quantity of Quantum Information
}

\author{
Vasil Penchev, vasildinev@gmail.com \\ Bulgarian Academy of Sciences: Institute of Philosophy and Sociology: \\ Dept. of Philosophy of Science
}

Abstract: The paper interprets the concept "operator in the separable complex Hilbert space" (particalry, "Hermitian operator" as "quantity" is defined in the "classical" quantum mechanics) by that of "quantum information". As far as wave function is the characteristic function of the probability (density) distribution for all possible values of a certain quantity to be measured, the definition of quantity in quantum mechanics means any unitary change of the probability (density) distribution. It can be represented as a particular case of "unitary" qubits. The converse interpretation of any qubits as referring to a certain physical quantity implies its generalization to non-Hermitian operators, thus neither unitary, nor conserving energy. Their physical sense, speaking loosely, consists in exchanging temporal moments therefore being implemented out of the space-time "screen". "Dark matter" and "dark energy" can be explained by the same generalization of "quantity" to non-Hermitian operators only secondarily projected on the pseudo-Riemannian space-time "screen" of general relativity according to Einstein's "Mach's principle" and his field equation.

Key words: quality, quantity, quantum information, qubit Hilbert space, space-time

\section{INTRODUCTION: “QUANTITY” IN QUANTUM MECHANICS VERSUS CLASSICAL PHYSICS}

At first glance, the concept of quantity in classical physics or experimental science at all does not seem to be linked to that of information (properly classical information, because that of quantum information has not appeared yet). However, the reverse perspective to scientific development can find that link as implicit there:

A bit of information, or an elementary and fundamental opposition corresponds unambiguously to any physical quantity: to be measured / not to be measured. However, a postulate for science to be ostensibly objective or "material" does not allow even for that implicit bit associable with any quantity to exist, but stating that value is always as "to be measured" including before measurement.

In fact, quantum mechanics was forced to reject that postulate as a prejudice since it should even generalize the bit at issue to a qubit therefore introducing the concept of quantum information as what is measured in qubits. The physical sense of the qubit consists in the following:

A probability density distribution (consequently normed in default) meaning a smooth "fan" of values for any quantity to be measured corresponds to it unambiguously before measurement. So, quantum mechanics made fun of the prejudice of classical science, which rejects that link of information, by transforming its negation in its main subject of research as a "quantum state" relevant to the measured physical quantity. 
However in the "classical" quantum mechanics, "quantity" has been defined partly in a compromising manner, "middle-of-the-road": indeed as a transformation of the probability (density) distribution, but allowing for the transformation to be only unitary and accordingly, the the corresponding operator defined on the separable complex Hilbert space of their characteristic functions to be only Hermitian. That restriction limits all admissible transformations, being interpreted physically, to those conserving energy, and thus, the quantity of time makes sense after them. In other words, time is saved not only as a fundamental physical quantity, but furthermore as an absolute condition for anything claiming to be physical or studible by physics.

The "slap glued on the chick" of the "classical" quantum mechanics due to that compromise is both "dark energy" and "dark matter", at that, about twenty times more tnah the "visible" ones in the universe. The magnitude of that "darkness" is the measure of our ignorance as well as for the falsity of human cognition, not only physical.

\section{QUANTUM QUANTITY AS INFORMATION BY QUANTUM INFORMATION}

The exhaustive reformulation of quantum mechanics as the theory of quantum information undertaken at the end of the last century allowed particularly for the compromise to "quantity" to be removed thoroughly in favor of quantum mechanics due to its "completeness' (including the corresponding theorems: Neumann 1932; Kochen, Specker 1967) as well as due to all phenomena of entanglement corroborated experimentally very well.

The concept itself of quantum information as a quantity measured in qubits allows for it to be considered as invariant to a new and generalized "quantum relativity", on the basis of which both entangled and non-entangled systems can be unified (Penchev 2021 June 8) unlike that of Hermititian operator relevant only to non-entangled systems therefore clearing the pathway for a new, more fundamental and properly informational understanding of "quantity", which can be illustrated unambiguously by a single qubit of any physical quality, respectively quantity:

$$
\begin{gathered}
\text { "Qubit" (def) } \Leftrightarrow \alpha \perp_{0}+\beta \perp_{1} \text { for: } \alpha, \beta \in C:|\alpha|^{2}+|\beta|^{2}=1 ; \perp_{0^{\prime}} \perp_{1} \subseteq H: \\
\left(\perp_{0}\right) \perp\left(\perp_{1}\right)
\end{gathered}
$$

Or written down by words: "Qubit" is any normed superposition by complex coefficients of two subspaces of the separable complex Hilbert space, orthogonal to each other, but not necessarily convex to each other (i.e. their intersection can be a nonempty set).

If a qubit is interpreted as any representation of a three-dimensional unit vector as the vector sum of two orthogonal two complex vectors, a bit of information is the particular case where the complenes of the two vectors are to be summed are whole positive numbers (that is: either " 0 " or "1").

The definition of quantity in the "classical" quantum mechanics as a unitary change of probability distribution or respectively, a Hermitiam operator can be rewritten by quantum information or by qubits in a few ways, one of which is the following: $\perp_{0^{\prime}} \perp_{1} \subseteq H$ are 
interpreted as relevant to state correspondingly "before" and "after" the Hermitian transformations:

Then, the condition of unitarity (i,e the operator to be Hermitian) can be visualized as the particular case of $\alpha, \beta \in C$ to be real numbers furthermore: a condition, which each qubit refers to a Hermitian operator ought to satisfy. The real axis implies a well-ordering, which can be "materialized" as a special physical quantity what time is, and naturally accompanied by the "conservation of energy conservation" in quantum mechanics (Penchev 2020 October 5).

If one considers the general case for the condition of unitarity not to be necessary, this corresponds for $\alpha, \beta \in C$ not to be necessarily real: the well-ordering of the real axis is cancelled, and the quantity of time is irrelevant in general. The definition of quantity after quantum information can be represented concisely: any qubit (rather than that obeys the condition of unitarity) can be a value of a quantity. If all quantities in classical physics as ratios of integers (since they are always rational numbers representable as ratios of integers), quantum mechanics after quantum information considers the generalized case where all quantities as relations (i.e. relevantly generalized "ratios") are qubits: the fundamental "unit" of any quantum quantity is a qubit.

\section{QUANTUM QUANTITY GENERALIZED BY QUANTUM INFORMATION}

There exists an implicit fundamental equation shared by any classical experimental science, which is not articulated, seeming to be trivial and absolutely obvious. However, it will be written expressly now, because quantum mechanics modifies it radically:

Physical quantity $=$ (Rational number)` multiplied by (the corresponding unit featuring the quality of the quantity)

For example, "A certain distance is 4.23 meters" means:

$$
\text { 'Distance }=(4.23) X(\text { the unit of meter }) \text { ' }
$$

This kind of representation is applicable to each physical quantity and each measurement.

At first glance, the generalization of quantum mechanics is not so radical, only substituting eventually the rational number (e.g. "4.23") by a relevant wave function; at that, any single measurement in quantum mechanics keeps the same form as a classical one.

However, the radicality becomes even shocking if one admits that "wave function" and the "quality" of the quantity at issue are complementary and thus interpretable as orthogonal to each other. In other words, the statement that the measuring wave function and the quality of the physical quantity constitutes in turn a pair, to which the concept and quantity of wave function are applicable: the corresponding quality of which is quantum information, and its unit is a qubit, therefore remaining physically dimensionless. Furthermore, quantum information can be considered not only as a universal physical substance more general than energy, but as the ultimate physical substance in virtue of the completeness of quantum mechanics (respectively, the "no hidden variables" theorems in it): indeed, the wave function measuring any physical 
quantity can be identified as the same with the meta-wave function able to represent the relation of the measuring wave function and the physical quantity at issue.

Furthermore, one can introduce the conservation of quantum information to express the above identification of the wave function on the level of physical quantity and it on the corresponding metalevel. The eventual and admissible, mutual transformations of "quantity" and "quality" (absolutely forbidden in classical science and even qualified as anti-scientific) obey quantum-information conservation, which regulates them excluding many classes of them as physically impossible. All phenomena of entanglement allow for such transformations between "quality" and "quantity". For example the change of any quantity due to entanglement can be interpreted as the transformation of the corresponding quality of that quantity into the change of wave function.

\section{NON-TEMPORAL BOTH QUANTUM MECHANICS AND PHYSICS}

The mutual transformations of "quantity" and "quality" cannot happen as processes in time and consequently they are not pictorable on the "temporal screen" of classical science or human empirical experience. The phrase "physical phenomena out of space-time" does not make sense for classical physics and even science: one of the most fundamental scientific postulates states that any natural phenomenon can happen only as a process in space-time.

Quantum mechanics weakens it, declaring that space-time is necessary only for measurement, which can really occur only in space-time, but the problem about its applicability to the physical phenomena by itself remains open: for example, "Copenhagen interpretation" suggests that the question does not sense therefore being a pseudo-problem reflecting only the prejudice of classical physics. Practically, Pauli's solution consisting in not assigning an operator to time (unlike all other physical quantities) or the rejection of the "four uncertainty" of energy and time by some authors (Broglie 1990; Mehra 1987; Costa de Beauregard 1986) follows the "Copenhagen" track (even rejecting it).

However, quantum correlations or the phenomena of entanglement confirmed experimentally (Camilleri 2009; Ladyman, Linnebo, Tomasz 2013; Cohen-Tannoudji, Diu, Laloë 2019) cannot fit in the paradigm of the "Copenhagen agnosticism" rather than only in that of classical physics, since what a quantum system is before measurement (i.e. "by itself" following Kant's idea of "Ding-an-sich") can be measured anyway as the change of probability distribution as a relation of at least two series of quantum measurements and situated at an arbitrary distance from each other. That change of probability distribution is not unitary definitively therefore suggesting that the intersection of the investigated remote probability distributions is not empty, though it should be such for, otherwise, it would imply the "spooky action at a distance" (in Einstein's eloquent enough metaphor) of entanglement.

Well, entanglement and thus quantum correlations are corroborated well enough nowadays: consequently there exists that "spooky action at a distance" (though rejected by Einstein in favor of the universality of space-time and implying the alleged "incompleteness of quantum mechanics": Einstein, Podolsky, Rosen 1935). 
Anyway, the violation of the "unconditional" space-time condition of classical physics seemed to be restricted only to quantum phenomena commensurable with the Planck constant and thus, not imposing any change of the fundamental understanding of science as it has been built relying on human empirical experience and only conservatively extended by relevant, though more and more complicated experiments during the period of classical science and physics and ended by the occurrence of quantum mechanics. In fact, the latter doubts the universal validity of "space-time screen" also to the macroscopic phenomena investigated by classical physics:

For example, the famous metaphor of "Schrödinger's cat" called to represent quantum phenomena by our usual experience utilizes the dissipative mechanism of many quantum measurements. It can be easily modified into two "entangled cats" situated anywhere in the universe interacting in virtue of quantum correlations and thus out of space-time: their fates will be entangled in a rigorous scientific meaning as the death of the one of them would change the chance of survival of the other instantaneously.

The converse implication can serve for confirming phenomena of macroscopic entanglement: if one observes any jump-like change in the probability distribution of any natural process which cannot be ascribed to any known cause, this can be explained in two alternative ways: (1) the action of some unknown cause yet not investigated by science; (2) macroscopic entanglement.

The peculiarity of the former hypothesis consists in its unfalsifiability (and consequently its non-scientificity in Popper's sense) if the latter conjecture is not researched: however, the law of teleportation needing two additional bits of classical information prevents its experimental corroboration (at first glance):

Alice's "Schrödinger's cat" and Bob's one are entangled, which is equivalent to the instantaneous transmission of a qubit between them. However, the fact that the former is observed to be either alive or dead is just that one classical bit of information transferable only in a subliminal channel. Bob waits for it. Meaning the distance to Alice, it calculates the corresponding moment in the past of his cat, but he is not able to implement any observation in a past moment, post factum

Nonetheless, an experiment able to confirm macroscopic entanglement of the remote cats seems to be possible. Bob and Alice conduct simultaneously two series of experiments on two sets of Schrödinger's cats, sending the results immediately. Waiting enough, each of them can notice the correlative coincidences just post factum in the corresponding probability distributions (if any). If those correlations violate the Bell (1964) equations (being a sufficient, but not necessary condition for entanglement), they would confirm macroscopic entanglement. If they do not violate them, both kinds of explanations remain possible ${ }^{1}$.

${ }^{1}$ The physical sense of violating the Bell equations consists in the following: those are correlations in the
separable complex Hilbert space rather than in space-time. So, their violation means immediately that
there exist corresponding physical phenomena, which are out of space-time. However, that corollary is so
shocking for the scientific common sense, that it dare not make it (just as all people who watch the "Nude
King", but dare not tell the truth). Any entangled wave functions are characteristic functions of
probability distributions which overlap each other by nonzero segments of the variable of the investigated 
One can visualize the direct transformation of "quality" and "quantity" by those two entangled Schrödinger cats considering the mutual correlations of the two bits "dead / alive" for each of both cats and granting for both "alive" and "dead" to be qualities. In each moment of time, the quality "dead" (respectively alive") is transformed in the quantity of a qubit, just as a quantity "transferred instantly" (or "properly mathematically"), and restored again by a correlative quality (i.e. properly physical) elsewhere. Particularly, classical physics suggests for itself, mathematics, and philosophy to be absolutely divided from each other, but quantum mechanics and information needs their unification, an expression of which is the mutual transformation of "quantity" and "quality".

The Schrödinger thought experiment suggests that the deterministic dissipative mechanism, which is able to unambiguously link quantum phenomena to macroscopic experience, is explicit. However, Bob and Alice might "bracket" it, admitting for that mechanism whether to be implicit, or even that it does not exist, therefore restricting themselves in a inherent "Copenhagen maner" to searching for coinciding simultaneous correlations in probability distribution of the same macroscopic phenomena distinguishing them from each other only in space-time localization. Such kind of "macroscopic quantum correlations" would confirm "macroscopic entanglement".

\section{A NON-TEMPORAL SOLUTION OF THE PROBLEM ABOUT "DARK MATTER" AND "DARK ENERGY"}

However, "macroscopic entanglement" definiable also experimentally as above would imply direct physical effects as in Alice's reference frame as in Bob's one not needing any communication between them, necessary for quantum correlations to be established experimentally:

The jump-like change of probability distribution observed by Bob or Alice implies a Hermitean operator, a physical quantity and a certain mass (respectively, energy) of some unknown something (which can be called "dark" metaphorically), to which that quantity due to the probability distribution change is to be ascribed. The total sum of all those changes once integrated all over the universe would result in "dark mass" and "dark energy" (independent of each other in virtue of the independence of mass and energy in general relativity and the Einstein field equation).

So, entanglement suggests to be an admissible hypothesis about the origin of "dark masss" and "dark energy" (Penchev 2021 October 20; Penchev 2021 March 9). According to it, they

or measured quantity. So, the necessary and sufficient condition of entanglement is: those probability distributions overlap each other, which is impossible in classical science for no probability distribution can correspond to any quantity in its framework, but only real and thus certain values; or by reflecting philosophically: entanglement refers to physical interaction of possibilities which cannot and will not be transformed in reality. They have to interact "in advance" (but "in advance" here means "before time or space-time to appear") to avoid any inconsistency in the ultimate solution what reality represents after decoherence and as a result of the "quantum calculation of the universe" (the quotation marks here are only an expression of diplomacy, tact, and courtesy). The ratio of dark matter and energy to visible ones $(\approx 20: 1)$ recalcultes quantum information into mass and energy unambiguously, therefore implicitly admitting their mutual transformation. 
would appear as "projections" of quantum information (being out of space-time by itself) on the space-time "screen", only on which general relativity is able to observe any physical phenomenon, however necessarily violating "Mach's principle" suggested by Einstein (1918):

it and postulatws that only energy and mass can be the source of gravitational field. On the contrary, quantum information turns out to be another source needing "dark mass" and "dark energy" to legitimize its existence since only the quantities of mass and energy are claimed to be admissible until now.

VI CONCLUSIONS, OR DARK MASS AND DARK ENERGY BY THE UNIFICATION AND MUTUAL TRANSFORMATION OF QUANTITY AND QUALITY

The sense of the paper consists in the interpretation of deducing the existence of "dark matter" and "dark matter" from the postulate of the totality (as in another paper: Penchev 2020 October 20) by the philosophical concepts of "quantity" and "quality" relying on the conception of "scientific transcendentalism" in turn interpreting "philosophical transcendentalism" in a falsifiable (thus non-metaphysical) way:

The area of quantity (e.g. understood in a contemporary and wider meaning as "mathematical structure") suggests the smooth transformation in its framework if and only if the quality of the quantity at issue remains the same. On the contrary, the area of quality supposes the discrete, jumplike transformation of any quality into another.

Quantum mechanics in physics and category theory in mathematics overcome or were forced to overcome that absolute opposition of quality and quantity featuring classical science and particularly as physics as mathematics motivated accordingly by the necessity: (1) to unify classical smooth physics and quantum phenomena to conserve quantum mechanics as an objective and experimental science; (2) to justify the consistent completeness of mathematics internally, i.e. admitting a mathematical proof of consistent completeness to be able to be demonstrated.

In the final analysis, the isomorphism of the consistent completeness of both quantum mechanics and category theory can be deduced relying as on the separable complex Hilbert space of quantum mechanics as on the space of all categories transformable between each other by functors in turn belonging to categories necessarily as the category of functors.

Both result into the qubit Hilbert space, which is able to deliver also a formal model of Husserl's phenomenology furthermore, therefore being able to unify (at worst, at least formally) a physical theory (such as quantum mechanics), a mathematical theory (such as category theory) and a philosophical doctrine (such as Hussel's phenomenology) thus all the three underlain by the same mathematical structure, namely the qubit Hilbert space.

The present paper is limited to the particular objectivity to explicate how the philosophical assumption of mutual direct transformation of quality and quantity implies the existence of dark matter and dark energy by the mediation of the qubit Hilbert space being furthermore in the foundations of the just cited three regions of cognition absolutely different according to classical science: 
If one admits quality and quantity to be transferable mutually, the quantitative (i.e. as a single quantity) expression of the nonzero area of both quantity and quality is meant necessarily in physics as dark matter and dark energy (also under the implicit condition for general relativity to be valid in order to be independent of each other):

Indeed, any quality in classical physics features a certain quantity furthermore needing to be a property of some physical entity belonging to this property. Being just physical, it shares a certain amount of energy (and an amount of mass being independent of the former amount of energy according to general relativity and e.g. obvious in the structure of the Einstein field equation) directly corresponding or being due to the quality of the property, the carrier of which is the entity with that energy:

If one changes the quality, this implies to be changed the entity and thus its energy (as the universal quantity shared by all physical entities) in general, resulting in the final analysis into the transformation of any quality represented in physics as a relevant quantity into an amount of the universal physical quantity of energy: thus being necessarily mapped on the absolute distinction of quality and quantity (in particular, "confessed" by classical science).

The change of the paradigm and corresponding worldview can be illustrated by the new gestalt for realizing the "Big Bang". All experimentally established quantitative properties and relations featuring the "first seconds of the universe after the Big Bang" are conserved, but interpreted otherwise: as the cumulative projection backwards in time of the entire decoherence, i.e. the quantity of decoherence determining a "field of decorence", further integrated throughout the space-time area of all the universe.

Though the two interpretations of the Big Bang are rather similar to each other, they can be distinguished experimentally anyway (and thus the one of them to be rejected in virtue of the factual mismatch). The explanation by the cumulative effect of the decoherence field implies a very small, but nonzero probability of "astronomical objects older than the universe" to be observed in virtue of quantum uncertainty since decoherence is a quantum phenomenon. That probability is smaller and smaller in the course of time featuring the "age of the universe", but remains enough to be discovered somewhere in the more and more expanding universe, especially in its "early stages" becoming observable only recently.

The new worldview, in which the transformation of quantity and quality is not only possible, but prevailing (e.g. in virtue of the predominance of dark matter and dark energy according to the experiments) seems so, figuratively:

A homogeneous "ocean" of quantum information being more general concept and physical quantity than space-time generates by itself and by mathematical necessity out of time, the space-time itself of the universe due to decoherence (e.g. representable as the process of dividing of the local and global space of the Standard model from each other): space-time is relevant only to the degree of absolute decoherence or respectively, to zero entanglement.

If one postulates space-time to be an absolute condition for anything claiming to be physical, that permanent process of decoherence (generating the space time itself) remains hidden and observable only: (1) as dark matter and dark energy; (2) particularly resulting in the concept of 
the "Big Bang" as a real phenomenon rather than a cumulative and probabilistic projection of all decoherence accompanying constality the generation of space-time.

In addition, that absolute understanding of the "Big Bang" implies an obvious inconsistency: itself is a huge violation of energy conservation, not only established as an absolute natural law after it, but necessary for it to be inferred interpolating the observed astronomical phenomena backwards in time: therefore relying on a "vicious circle" logically.

\section{References:}

Bell, J. S. (1964) “On the Einstein Podolsky Rosen Paradox" Physics (New York) 1 (3): 195-200, . https://journals.aps.org/ppf/abstract/10.1103/PhysicsPhysiqueFizika.1.195.

de Broglie, L. (1990) Heisenberg's Uncertainties and the Probabilistic Interpretation of Wave Mechanics: with Critical Notes of the Author (Series: Fundamental Theories of Physics 40). Dordrecht / Boston / London: Kluwer (Springer Netherlands). Chapter 9 "'Heisenberg's Fourth Uncertainty Relation," pp. 103-123.

Camilleri, K. (2009) "A history of entanglement: Decoherence and the interpretation problem," Studies in History and Philosophy of Science Part B: Studies in History and Philosophy of Modern Physics 40 (4): 290-302.

Cohen-Tannoudji, C., B. Diu, F. Laloë (2019) Quantum Mechanics. Volume 3: Fermions, Bosons, Photons, Correlations, and Entanglement. Weinheim (Germany): Wiley-VCH (Chapter XXI "'Quantum entanglement, measurements, Bell's inequalities") pp. 2187-2264.

Costa de Beauregard, O. (1986) "Bohr's discussion of the fourth uncertainty relation revisited," Foundations of Physics $\mathbf{1 6}$ (9): 937-939.

Einstein, A. (1918) "Prinzipielles zur allgemeinen Relativitätstheorie," Annalen der Physik 55 (4): $241-244$, https://onlinelibrary.wiley.com/doi/abs/10.1002/andp.19183600402.

Einstein, A, B. Podolsky, N. Rosen (1935) "Can Quantum-Mechanical Description of Physical Reality Be Considered Complete?" Physical Review 47 (10): 777-780

https://journals.aps.org/pr/abstract/10.1103/PhysRev.47.777.

Kochen, S., E. P. Specker (1967) "The problem of hidden variables in quantum mechanics," Journal of Mathematics and Mechanics 17 (1): 59 - 87, http://www.iumj.indiana.edu/IUMJ/fulltext.php?artid=17004\&year=1968\&volume=17 .

Ladyman, J., Ø. Linnebo, B. Tomasz (2013) "Entanglement and non-factorizability," Studies in History and Philosophy of Science Part B: Studies in History and Philosophy of Modern Physics 44 (3): $215-221$.

Mehra, J. (1987) "Niels Bohr's discussions with Albert Einstein, Werner Heisenberg, and Erwin Schrödinger: The origins of the principles of uncertainty and complementarity," Foundations of Physics 17 (5): $461-506$.

von Neumann, J. (1932) Mathematische Grundlagen der Quantenmechanik. Berlin: J. Springer, pp. 167-173, https://gdz.sub.uni-goettingen.de/id/PPN379400774.

Penchev, V. (2021 June 8) "The Symmetries of Quantum and Classical Information. The Resurrected 'Ether' of Quantum Information,” SSRN, https://dx.doi.org/10.2139/ssrn.3861105 or https://papers.ssrn.com/sol3/papers.cfm?abstract id=3861105 .

Penchev, V. (2021 March 9) "The Generalization of the Periodic Table: The 'Periodic Table' of 'Dark Matter'," SSRN $\quad$ https://papers.ssrn.com/sol3/papers.cfm?abstract $\mathrm{id}=3800823$ or https://dx.doi.org/10.2139/ssm.3800823.

Penchev, V. (2020 October 20) "Two deductions: (1) from the totality to quantum information conservation; (2) from the latter to dark matter and dark energy," SSRN, or https://papers.ssrn.com/sol3/papers.cfm?abstract id=3683658 https://dx.doi.org/10.2139/ssrn.3683658 . 
Penchev, V. (2020 October 5) "Quantum-Information Conservation. The Problem About 'Hidden Variables', or the 'Conservation of Energy Conservation' in Quantum Mechanics: A Historical Lesson for Future Discoveries," SSRN, https://dx.doi.org/10.2139/ssrn.3675319 or https://papers.ssrn.com/sol3/papers.cfm?abstract id=3675319 . 